\title{
Gastrointestinal Illness Associated with Rancid Tortilla Chips at a Correctional Facility - Wyoming, 2015
}

\author{
Tiffany Lupcho, $\mathrm{MPH}^{1}$; Alexia Harrist, $\mathrm{MD}, \mathrm{PhD}^{1,2}$; Clay Van Houten, $\mathrm{MS}^{1}$
}

On October 12, 2015, a county health department notified the Wyoming Department of Health of an outbreak of gastrointestinal illness among residents and staff members at a local correctional facility. The majority of ill persons reported onset of symptoms within 1-3 hours after eating lunch served at the facility cafeteria at noon on October 11 . Residents and staff members reported that tortilla chips served at the lunch tasted and smelled like chemicals. The Wyoming Department of Health and county health department personnel conducted case-control studies to identify the outbreak source. Consuming lunch at the facility on October 11 was highly associated with illness; multivariate logistic regression analysis found that tortilla chips were the only food item associated with illness. Hexanal and peroxide, markers for rancidity, were detected in tortilla chips and composite food samples from the lunch. No infectious agent was detected in human stool specimens or food samples. Extensive testing of lunch items did not identify any unusual chemical. Epidemiologic and laboratory evidence implicated rancid tortilla chips as the most likely source of illness. This outbreak serves as a reminder to consider alternative food testing methods during outbreaks of unusual gastrointestinal illness when typical foodborne pathogens are not identified. For interpretation of alternative food testing results, samples of each type of food not suspected to be contaminated are needed to serve as controls.

Wyoming Department of Health investigators were notified that a total of 16 residents and staff members at a local mixed-sex correctional facility were evaluated at the facility's medical office on October 11, 2015, after reporting stomach cramping, gas, bloating, diarrhea, and burping. Active case finding was conducted during October 12-28, using a standardized questionnaire administered by telephone or in-person, or self-completed.

Because facility residents were continually being admitted and released, investigators could not assess exposures in the entire population at the time of the outbreak; therefore, an initial case-control study was used to identify specific meals and food items associated with illness. A case was defined as the onset of nausea, vomiting, stomach cramps, diarrhea, gas, or bloating in any facility resident or staff member during October 9-12. Controls were defined as residents or staff members who consumed food from the facility cafeteria during October 8-11 and did not report any of these symptoms. To substantiate the link between consumption of one food item and illness, investigators performed a nested case-control study focusing on persons who became ill with more severe symptoms on or after October 11. A case of severe illness was defined as the occurrence of vomiting or diarrhea in any facility resident or staff member during October 11-12. Controls were defined as residents or staff members who consumed meals from the facility cafeteria during October 10-11 and did not experience any illness. The age and resident status of case-patients and controls were compared using the Mann-Whitney $U$ test and Fisher's exact test, respectively.

Meals served at the correctional facility during October 8-11 were included in univariate analyses. Meals significantly associated with illness in univariate analyses were included in multivariate logistic regression models. Stool specimens were collected from four case-patients and tested by the Wyoming Public Health Laboratory for enteric pathogens. Because samples of food items served at every meal were frozen and stored by the correctional facility for an extended period of time, investigators were able to obtain frozen samples of all food items served at the October 11 lunch meal for testing. The Wyoming National Guard's 84th Civil Support Team examined frozen food samples using gas chromatography-mass spectrometry to assess for possible chemical contamination or unusual added substances. A private food testing laboratory tested a frozen mixture of beef and beans, nacho cheese sauce, tortilla chips, and Spanish rice (composite sample) for bacterial toxins and peroxide levels.

At the time of investigation, there were an estimated 254 residents and 75 staff members at the facility. The questionnaire response rate among residents was $62 \%$ (157 of 254) and among staff members was 84\% (63 of 75). Overall, 220 $(67 \%)$ of the 329 facility residents and staff members completed the questionnaire; 109 (33\%) were unavailable or did not participate. During in-person interviews, residents reported that tortilla chips served at lunch on October 11 tasted and smelled like chemicals. Although the tortilla chips reportedly smelled and tasted foul, many persons consumed them.

Among 220 persons interviewed, 133 (60\%) met eligibility for the initial case-control study with 79 case-patients and 64 controls identified. The median age of the case-patients ( 30 years; range $=20-77$ years) was slightly less than that of the controls (36 years; range $=19-63$ years $)(\mathrm{p}=0.02)$. The percentage of residents among case-patients (76 of $79,96 \%$ ) was similar to 
the percentage among controls (59 of 64, 92\%) ( $\mathrm{p}=0.47$ ). Among case-patients, the predominant symptoms reported were nausea ( 65 of $79,82 \%)$, gas/bloating ( 61 of $79,77 \%$ ), stomach cramps ( 59 of $79,75 \%$ ), and diarrhea (57 of $79,72 \%$ ); a smaller number reported vomiting (17 of 79, 21\%). Most case-patients experienced short-lived illness and recovered fully with a median illness duration of 24.5 hours (range $=2$ hours -14 days). More than half of the case-patients with known illness onset times ( 48 of $78,62 \%$ ) became ill within 1-3 hours after eating lunch on October 11 , indicating that the outbreak likely was caused by a point source exposure (Figure).

Lunch on October 11 was the only meal significantly associated with illness in multivariate analysis (adjusted odds ratio $=22.8$ ) (Table 1). Food items served included nacho cheese sauce, tortilla chips, beef and beans, Spanish rice, salad, ranch dressing, a cookie, and multiple drink options. Certain food items served at the meal were often consumed together (e.g., tortilla chips and nacho cheese sauce) and associated with illness in univariate analysis; these items were included in multivariate modeling. Tortilla chips were the only food item associated with illness in multivariate analysis (adjusted odds ratio $=9.7)($ Table 2$)$.

A total of 55 case-patients and 57 controls were identified for the nested case-control study. The median age among case-patients ( 30.5 years; range $=20-77$ years) was similar to that of controls (median $=35.5$ years; range $=19-63$ years) $(p=0.07)$. No difference in the percentage of residents among case-patients ( 52 of 55; 95\%) and controls ( 56 of 57; 98\%) was observed ( $\mathrm{p}=0.36$ ). Multivariate modeling identified the lunch on October 11 (adjusted odds ratio $=10.5 ; 95 \%$ confidence interval $[\mathrm{CI}]=1.2-90.1)$ and tortilla chips (adjusted odds ratio $=7.9 ; \mathrm{CI}=1.4-45.3$ ) as exposures associated with illness.

No enteric pathogens were identified in stool specimens tested by the Wyoming Public Health Laboratory, and no bacterial toxins were detected in a composite sample of food items served at the October 11 lunch meal. Because no infectious source was identified and epidemiologic association of tortilla chips with illness existed, investigators tested the tortilla chips for chemicals to identify potential contamination. On October 30, testing of frozen samples of tortilla chips, nacho cheese sauce, and a composite food mixture (beef, beans, nacho cheese sauce, tortilla chips, and Spanish rice) by the Wyoming National Guard's 84th Civil Support Team for possible chemical contamination did not yield any unusual chemicals; however, hexanal, which is used as a measure of rancidity $(1,2)$, was detected in the tortilla chip sample. On December 10, 2015, the private food testing laboratory measured the peroxide value, another marker for rancidity, in the composite sample of frozen lunch items. The peroxide value of the composite food sample was $377 \mathrm{meq} / \mathrm{kg}$. Laboratory staff members reported that the peroxide value of the composite food sample was markedly high, but they could not provide any reference ranges because of a lack of food not suspected to be contaminated

FIGURE. Number of residents and staff members $\left(\mathrm{N}=79^{*}\right)$ at a correctional facility reporting gas, bloating, abdominal cramps, diarrhea, nausea, or vomiting, by time of onset of first symptom ${ }^{\dagger}$ - Wyoming, October 10-12, 2015

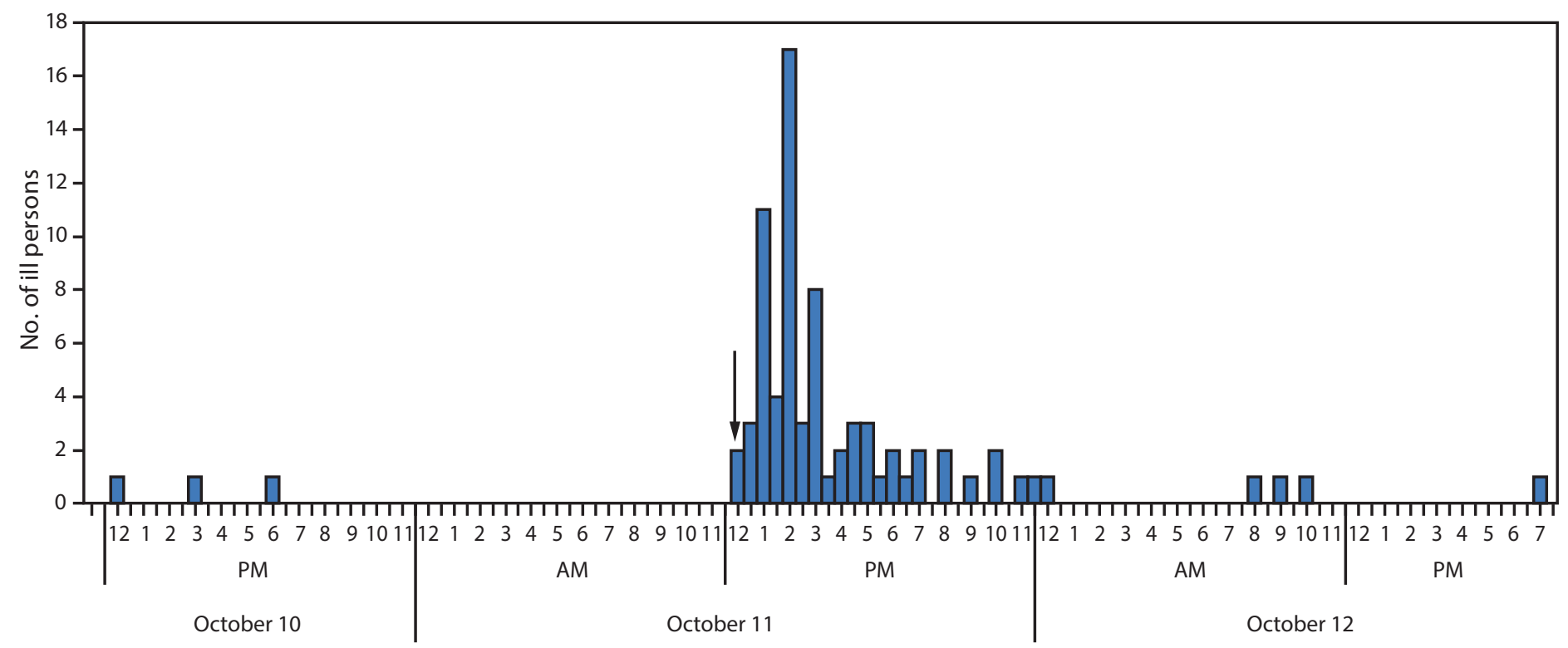

Time of day

* Onset time was unavailable for one patient.

${ }^{\dagger}$ Lunch was served at noon on October 11. 
TABLE 1. Univariate and multivariate logistic regression analysis of meal exposures among 79 case-patients and 64 controls in an outbreak of gastrointestinal illness at a correctional facility - Wyoming, October 2015

\begin{tabular}{|c|c|c|c|c|c|c|c|c|}
\hline Meal & Date & $\begin{array}{c}\text { No. } \\
\text { exposed }\end{array}$ & $\begin{array}{l}\text { No. of case-patients } \\
\text { reporting exposure* } \\
\text { (\%) }\end{array}$ & $\begin{array}{l}\text { No. of controls } \\
\text { reporting exposure* } \\
\text { (\%) }\end{array}$ & OR (95\% Cl) & p-value ${ }^{\dagger}$ & $\begin{array}{l}\text { Adjusted OR } \\
(95 \% \mathrm{Cl})\end{array}$ & $p$-value ${ }^{\S}$ \\
\hline Breakfast & $10 / 8 / 15$ & 137 & $52 / 75(69)$ & $38 / 62(61)$ & $1.4(0.7-2.9)$ & 0.37 & - & - \\
\hline Lunch & $10 / 8 / 15$ & 136 & $59 / 74(80)$ & $40 / 62(64)$ & $2.2(1.0-4.7)$ & 0.05 & $0.9(0.3-3.5)$ & 0.96 \\
\hline Dinner & $10 / 8 / 15$ & 132 & 66/74 (89) & $41 / 58(71)$ & $3.4(1.3-8.6)$ & 0.01 & $0.9(0.2-4.6)$ & 0.92 \\
\hline Breakfast & $10 / 9 / 15$ & 136 & $52 / 76(68)$ & $41 / 60(68)$ & $1.0(0.5-2.1)$ & 1.00 & - & - \\
\hline Lunch & $10 / 9 / 15$ & 135 & $65 / 75$ (87) & $43 / 60(72)$ & $2.6(1.1-6.1)$ & 0.05 & $0.5(0.1-2.3)$ & 0.43 \\
\hline Dinner & 10/9/15 & 137 & 65/76 (85) & 39/61 (64) & $3.3(1.5-7.6)$ & $<0.001$ & $2.5(0.6-10.8)$ & 0.21 \\
\hline Breakfast & $10 / 10 / 15$ & 138 & $41 / 78$ (52) & $30 / 60(50)$ & $1.1(0.6-2.2)$ & 0.86 & - & - \\
\hline Lunch & $10 / 10 / 15$ & 136 & $64 / 75$ (85) & $42 / 61(69)$ & $2.6(1.1-6.1)$ & 0.02 & $1.2(0.3-4.5)$ & 0.83 \\
\hline Dinner & $10 / 10 / 15$ & 140 & $66 / 78(85)$ & $47 / 62(76)$ & $1.7(0.7-4.1)$ & 0.20 & - & - \\
\hline Breakfast & $10 / 11 / 15$ & 140 & $36 / 77$ (47) & $33 / 63(52)$ & $0.8(0.4-1.5)$ & 0.61 & - & - \\
\hline Lunch & $10 / 11 / 15$ & 141 & 78/79 (99) & $46 / 62(74)$ & $27.1(3.4-211.3)$ & $<0.001$ & $22.8(2.5-209.5)$ & 0.01 \\
\hline
\end{tabular}

Abbreviations: $\mathrm{Cl}=$ confidence interval; $\mathrm{OR}=$ odds ratio.

* Not all of those interviewed answered each question.

† Fisher's exact test two-sided statistic.

$\S$ Chi-square statistic.

but produced at approximately the same time to serve as control samples. The tortilla chips were purchased from a food distribution center, and the production and expiration dates were unknown. All remaining bags of tortilla chips were discarded before investigators could obtain them for testing.

\section{Discussion}

This report describes a point source outbreak of gastrointestinal illness at a correctional facility where no infectious etiology was identified, but epidemiologic evidence implicated a single food item, tortilla chips, as the source of illness. Hexanal is a compound common in food additives, dyes, and insecticides. However, both hexanal and peroxide are markers of rancidity $(1,2)$. The high peroxide value in the composite food sample and detection of hexanal in the tortilla chips indicate the chips might have been rancid. Rancidity results from degradation of oils and fats, a process that can occur through exposure to heat and light, and can affect the taste and quality of food. The foul taste and odor of the tortilla chips reported by facility residents and staff members further support this hypothesis. However, because approximately 3-8 weeks had elapsed between the date of food service and testing, the rancidity of the chips at the time of service could not be confirmed. Interpretation of food testing results from this outbreak was difficult because no food control samples for each food item tested were available for comparing results. For example, comparing the peroxide and hexanal levels from the suspect food that was served with those of tortilla chips not suspected to be contaminated and produced by the same manufacturer on approximately the same date would have allowed investigators to better determine what levels would be expected from exposure to heat and light over time and what levels might be associated with adverse health events.

Outbreaks caused by intentional and unintentional chemical contamination of food (e.g., with pesticides and ammonia) have been described (3-7) and are characterized by a rapid onset of illness. Food testing in this outbreak, however, did not detect evidence of adulteration or added chemicals that could explain the increase in gastrointestinal illness after consumption of tortilla chips. Further, in outbreaks caused by chemical contamination, persons with illness typically experience nausea, vomiting, and neurologic symptoms (3-7); case-patients in this outbreak did not report neurologic symptoms, and only $21 \%$ reported vomiting. Instead, $>70 \%$ of case-patients reported nausea, burping, gas, or diarrhea. 


\section{Summary}

What is already known about this topic?

Although consumption of rancid food can cause gastrointestinal illness, few outbreaks have been documented.

What is added by this report?

In October 2015, an outbreak of gastrointestinal illness occurred at a Wyoming correctional facility. Epidemiologic and laboratory evidence implicated rancid tortilla chips as the likely source of illness.

What are the implications for public health practice?

The likelihood of rancid tortilla chips as the source of illness in this outbreak serves as a reminder to consider alternative sources of illness other than foodborne pathogens during outbreaks of unknown gastrointestinal illness. When rancidity is suspected as the source of illness, specific food testing methods are needed that might not be readily available at state public health laboratories.

Few outbreaks of gastrointestinal illness associated with consumption of rancid food have been documented (8). Rancidity was identified as the source of an outbreak in India in which 80 persons became ill with abdominal cramping, vomiting, and diarrhea within 1.5-2 hours after consuming rancid biscuits (8). The biscuits were deemed rancid through peroxide testing. The Wyoming correctional facility outbreak illustrates the importance of considering noninfectious etiologies of illness and collecting all suspected foods, as well as samples not suspected to be contaminated to serve as controls, to ensure that food testing can be fully interpreted. When considering rancidity as a source of illness, specific testing methods not routinely available or performed at public health laboratories are needed.

\section{Acknowledgments}

Casper-Natrona County Health Department; Wyoming Public Health Laboratory; Wyoming National Guard's 84th Civil Support Team; Cody Loveland, Katie Bryan, Wyoming Department of Health.

${ }^{1}$ Wyoming Department of Health; ${ }^{2}$ Epidemic Intelligence Service, CDC.

Corresponding author: Tiffany Lupcho, tiffany.lupcho@wyo.gov, 307-777-7007.

\section{References}

1. Hu M, Jacobsen C, editors. Oxidative stability and shelf life of foods containing oils and fats. San Diego, CA: Academic Press, AOCS Press; 2016.

2. NP Analytical Laboratories. Measuring rancidity in fats and oils. St. Louis, MO: NP Analytical Laboratories. https://www.npal.com/docs/npal_ document_71.pdf

3. Anderson S, DeMent J, Banez Ocfemia C, Hunt D. Outbreaks of methomyl poisoning caused by the intentional contamination of salsa at the Mi Ranchito restaurant in Lenexa, KS-August 2009. Topeka, KS: Kansas Department of Health and Environment; 2011. http://www. kdheks.gov/epi/download/Final_Mi_Ranchito_Report.pdf

4. CDC. Aldicarb as a cause of food poisoning-Louisiana, 1998. MMWR Morb Mortal Wkly Rep 1999;48:269-71.

5. CDC. Multiple outbreaks of gastrointestinal illness among school children associated with consumption of flour tortillas-Massachusetts, 20032004. MMWR Morb Mortal Wkly Rep 2006;55:8-11.

6. CDC. Endrin poisoning associated with taquito ingestion-California. MMWR Morb Mortal Wkly Rep 1989;38:345-7.

7. Dworkin MS, Patel A, Fennell M, et al. An outbreak of ammonia poisoning from chicken tenders served in a school lunch. J Food Prot 2004;67:1299-302.

8. Bhat RV, Vemula SR, Pokkunuri Y, Siddula G, Purnachandra GK. Foodborne disease outbreak due to consumption of rancid biscuits. J Toxicol Clin Toxicol 1995;33:219-22. http://dx.doi.org/10.3109/15563659509017987 